\title{
EL ESPACIO AÉREO Y SU REGLAMENTO INTERNACIONAL
}

\author{
THE AIRSPACE AND ITS INTERNATIONAL REGULATIONS \\ Manuel Ángel Calvo Castillo* \\ Escuela Superior de Guerra, México \\ macc_235@hotmail.com
}

\section{RESUMEN:}

En el artículo 42 Constitucional de nuestra carta magna establece que el espacio aéreo es parte fundamental de la integración del Estado Mexicano, toda vez que es parte integral del territorio nacional que comprende “... VI. El espacio situado sobre el territorio nacional, con la extensión y modalidades que establezca el propio derecho internacional y su correspondiente soberanía pero que, además, posea capacidades de proyección a nivel regional, hemisférico y global..." de lo anterior se desprende una normatividad fundamental que por ser muy especializada es poco conocida, al ser un sector tan importante y estratégico en el ámbito internacional y nacional es procedente identificar cuáles son algunas de los principales regímenes jurídicos de este sector tan importante.

\section{Palabras clave:}

Espacio aéreo, Derecho Aeronáutico, Derecho Espacial, Soberanía, Normatividad aeronáutica.

\section{ABSTRACT:}

In Article 42 of the Constitution of our Magna Carta establishes that airspace is a fundamental part of the integration of the Mexican State, since it is an integral part of the national territory that includes "... VI. The space located on the national territory, with the extension and modalities established by international law itself and its corresponding sovereignty, but which, in addition, has projection

* Doctor en Administración Pública, docente de la Escuela Superior de Guerra y del Heroico Colegio Militar. 
capabilities at the regional, hemispheric and global levels... "From the above, a fundamental regulation follows that Because it is highly specialized, it is little known, as it is such an important and strategic sector at the international and national level, it is appropriate to identify which are some of the main legal regimes of this very important sector.

\section{Keywords:}

Airspace, Aeronautical Law, Space Law, Sovereignty, Aeronautical Regulations.

Espacio y tiempo son dos nociones estrechamente vinculadas que tienen el carácter de ideas trascendentales. Constituyen una condición a priori universal, necesaria a todo conocimiento. Originariamente son datos intuitivos, subjetivos, a partir de los cuales podemos construir nociones de espacio y tiempo objetivas atribuyéndoles una realidad exterior a nuestra conciencia. Como realidad perceptible por los sentidos, nos encontramos ante la imposibilidad de definir al espacio.

Con respecto al espacio aéreo una disquisición común es la de diferenciar aire de espacio aéreo. El aire posee una naturaleza etérea, fluida, inmensa y móvil que determina su imposibilidad de aprehensión física. Ya los romanos lo consideraban res communis omnis ${ }^{1}$ y vendría a constituir un contenido. El espacio aéreo es un continente cuyo contenido es la atmósfera terrestre. Lo cierto es, que el espacio aéreo es un ámbito en el que se desarrollan actividades humanas, siendo la aviación una de ellas.

El espacio aéreo no es un bien, porque no es físicamente apropiable, pero se considera al espacio aéreo como un bien o cosa, que tiene una medida de valor, y es susceptible de propiedad, y pasible objeto de soberanía. En general es aceptado el hecho de que todo Estado como se sabe se compone de población, territorio, gobierno y orden jurídico.

Es comprobable que a lo largo de la historia, el escenario en que se ha movido el Estado ha ido cambiando, es así que de un territorio unidimensional que caracteriza a la llamada ciudad-Estado, se pasa al territorio bi-dimensional, cuando el mar deja de ser considerado res nulius ${ }^{2}$ es a fines del siglo XVIII (1782) cuando aparecen los globos de aire caliente, los artefactos de los hermanos Montgolfier $^{3}$ que comienza a surgir la idea de dominio vertical de los Estados, y se empieza a hablar de territorio tridimensional (tierra-aire-mar).

Surgen entonces los primeros problemas jurídicos de cómo regular la conducta del hombre y los derechos soberanos y jurisdiccionales de los Estados.

1 La res communes omnium está formada por aquéllas cosas que por naturaleza están destinadas al uso de todos los hombres y que, por tanto, no podrían pertenecer a un solo individuo; incluye entre las cosas comunes a todos por derecho natural: el aire (air), el agua corriente (agua profluens), el mar (mare) y, consiguientemente, el litoral del mar (et per hoc litora maris).

2 Cosa de nadie; es decir, cosa sin dueño. Es la protagonista objetiva de la ocupación como forma de adquirir la propiedad. Los objetos arrojados al mar o los que las olas depositan en las playas, y con independencia de su naturaleza física.

3 Joseph-Michel Montgolfier y Jacques-Étienne Montgolfier fueron dos hermanos franceses conocidos por ser considerados los inventores del globo aerostático. 
Finalmente, con el advenimiento de la era espacial aparece lo que se ha dado en llamar el territorio cuatridimensional, agregándose a los demás ámbitos el espacio ultraterrestre.

El tratamiento del espacio aéreo y su régimen jurídico tiene connotaciones que involucran al Derecho Público, al Derecho Privado con temas como la extensión de los derechos del propietario del suelo sobre el espacio aéreo que se encuentra sobre sus fundos o las limitaciones al derecho de propiedad en beneficio de la seguridad y regularidad del transporte aéreo, existiendo ciertas áreas que tocan ambas ramas del Derecho.

\section{DELIMITACIÓN ENTRE EL ESPACIO AÉREO Y EL ESPACIO ULTRATERRESTRE}

Los Estados reconocen la existencia de un espacio aéreo y de un espacio ultraterrestre, que si bien, han sido regulados internacionalmente, no se ha llegado a determinar precisamente cuáles son sus límites físicos. ${ }^{4} \mathrm{La}$ importancia de la delimitación entre ambos espacios reside en la diversidad de sus regímenes jurídicos. En efecto, mientras en el espacio aéreo impera el principio de soberanía, en el espacio ultraterrestre predomina el principio de libertad. El espacio aéreo está sometido a soberanía estatal completa y exclusiva, mientras que el espacio ultraterrestre no. ${ }^{5}$

La falta de una definición o delimitación del espacio aéreo puede dar lugar a una incertidumbre jurídica respecto al derecho a aplicar: el Derecho Aeronáutico o el Derecho Espacial. Ni la Convención de París de 1919, ni el Convenio sobre Aviación Civil Internacional (Convenio de Chicago de 1944) definen o señalan los límites de soberanía o las fronteras. Así el Artículo $1^{\circ}$ del Convenio sobre Aviación Civil Internacional (Convenio de Chicago de 1944 ), ratificado por nuestro país establece la soberanía plena y exclusiva de los Estados en el espacio aéreo situado sobre su territorio, mientras que el Artículo $2^{\circ}$ del citado instrumento se refiere a los límites horizontales de la soberanía estatal, pero no a los verticales. ${ }^{6}$

Tampoco el Tratado sobre los principios que gobiernan las actividades de los Estados en la exploración y utilización del espacio ultraterrestre, incluso la luna y otros cuerpos celestes, conocido como Tratado del espacio de 1967, delimita el espacio aéreo, ya que no se definen los límites del espacio ultraterrestre. Desde 1983, el tema de la delimitación entre ambos espacios, viene siendo estudiado en la Comisión sobre la Utilización del Espacio Ultraterrestre con Fines Pacíficos (COPUOS por sus siglas en inglés). ${ }^{7}$

\footnotetext{
4 ORTIZ AHLF, Loretta, Derecho Internacional Público, University Press Oxford, tercera edición, México, 2004, pp. 105-108.

Cfr. El Espacio Aéreo y su Régimen Jurídico, http://bit.ly/2aabey5, 03/05/2016, 08:27.

Cfr. Convenio sobre Aviación Civil Internacional (convenio de Chicago), http://bit.ly/2a5tKqa, 03/05/2016, $11: 43$.

7 Cfr. COPUOS, http://www.unoosa.org/pdf/transcripts/copuos/COPUOS, 03/05/2019, 11:30.
} 
Ese año, la delegación rusa presentó una propuesta en la que propuso que:

... el límite entre el espacio atmosférico y el espacio ultraterrestre se determina (se) por acuerdo entre los Estados a una altura no superior a $110 \mathrm{kms}$ sobre el nivel del mar y se reafirma (se) jurídicamente mediante la concertación de un instrumento de derecho internacional de carácter obligatorio...

... En este instrumento se preverá también que se preservará el derecho de paso inocente con fines pacíficos de un objeto espacial de cualquier Estado sobre el territorio de otros Estados a alturas inferiores al límite convenido al salir de su órbita y reingresar a la tierra...8

La Sub-Comisión de Asuntos Jurídicos de dicho organismo, en su $43^{\circ}$ período de sesiones celebrado en Viena del 29 de marzo al 8 de abril de 2004, fue de la opinión de que la definición y delimitación del espacio ultraterrestre continuaba siendo un tema importante, en consideración de la existencia de diferentes regímenes jurídicos y de los continuos avances tecnológicos, teniendo en cuenta los principios de soberanía y libertad que los diferencia. ${ }^{9}$ Doctrinariamente se han establecido límites al espacio aéreo y al espacio ultraterrestre en base a criterios técnicos y científicos, los cuales han fracasado al igual que los criterios jurídico-políticos a los que se arribaría mediante una delimitación convencional.

Los criterios técnicos o científicos más conocidos se basan en la composición de la atmósfera, la densidad del aire como elemento de sustentación de las aeronaves, la delimitación tomando en cuenta la órbita de un satélite artificial de la tierra, la Línea Von Karman (100-118 km de altura) y la delimitación en función de los efectos de la gravedad, entre otros. ${ }^{10}$ La fijación de una altura para ubicar el plano divisorio entre los dos espacios irá cambiando con los adelantos tecnológicos, salvo que se tome una altura determinada y se resuelva por la vía convencional.

Al no existir criterios tecnológicos y científicos uniformes, y dado que los mismos no brindaban certidumbre en la fijación de la frontera, muchos autores desecharon esta forma de delimitación y se inclinaron a buscar una solución normativa, intentando lograr un criterio jurídico de delimitación por la vía de un acuerdo internacional.

Para los criterios jurídico-políticos, lo importante no son tanto los límites entre los espacios aéreo y ultraterrestre, sino la extensión de la soberanía en sentido vertical, tendiendo a determinar el concepto de frontera aérea en relación con el principio de soberanía de los Estados en el espacio aéreo. ${ }^{11}$ Las posiciones relacionadas con la extensión del poder estatal respecto del espacio aéreo van desde la tesis de la soberanía extendida hasta al infinito llegando a aquella que se inclina por la abolición del principio de soberanía, ya que entiende que la navegación

$8 \quad C f r$. Tratados y principios de las Naciones Unidas sobre el Espacio Ultraterrestre, ONU, http://bit.ly/2a0yYlD, 03/05/2019, 13:39.

9 Cfr. Comisión sobre la Utilización del Espacio Ultraterrestre con Fines Pacíficos, http://bit.ly/2am29SM, 03/05/2019, 19:08.

10 Zona o línea imaginaria con la que se trata de señalar el límite entre el espacio aéreo y el espacio exterior.

11 Dr. LINARES, Antonio, Régimen Jurídico del Espacio Aéreo, http://bit.ly/2a9hcw9, 03/05/2016, 22:49. 
aérea exige la necesidad de la libertad del aire, puesto que a medida que nacen nuevos Estados, se multiplican los espacios aéreos sometidos a soberanía, lo que complicaría la navegación aérea en cuanto se negasen permisos de sobrevuelo que obligarían a las aeronaves a tomar otras rutas.

Otras teorías se refieren a la posibilidad de control efectivo del espacio aéreo, a la limitación de lo necesario para la seguridad y defensa del Estado o la delimitación del espacio en zonas. En general la posición dominante se inclina por una regulación convencional que establezca una seguridad jurídica. ${ }^{12}$ Pero aquí el tema se complica al momento de decidir entre los criterios espaciales y los funcionales. El enfoque espacial establecería dos zonas, cuyo límite sería una altitud de $100 \mathrm{o}$ 118 kilómetros por encima del nivel del mar. Hasta los 100 o 118 kms, se aplicaría el principio de soberanía, luego el de libertad en el espacio ultraterrestre. ${ }^{13}$

Sin embargo, los problemas subsisten, ya que un mismo objeto espacial tendría en un tiempo brevísimo regulaciones jurídicas diferentes. De adoptarse el criterio funcional, lo relevante, sería el tipo de actividad desarrollada: aérea o espacial. ${ }^{14}$ Aun así, los problemas se mantienen en vista de las diferentes posiciones políticas entre los Estados: las grandes potencias espaciales son partidarias de la mayor libertad, mientras que los Estados cuya participación en la carrera espacial es menos activa, se oponen a una libertad irrestricta que pueda conducir a una amenaza a la soberanía aérea.

Numerosos Estados han formulado reivindicaciones de soberanía sobre segmentos del espacio que se encuentran por encima de sus territorios, lo que frustra los intentos de definir los límites entre el espacio aéreo y el ultraterrestre.

\section{LA SOBERANÍA SOBRE EL ESPACIO AÉREO}

Cuando nace la idea de dominio sobre el espacio aéreo los conocimientos cósmicos de entonces, nos enfrentan a una idea de espacio distinta de la actual. Debido a la naturaleza especial del espacio aéreo, hay quienes consideran que debería hablarse de jurisdicción aérea (imperium) ${ }^{15}$ antes que de soberanía (dominium $)^{16}$ cuando comenzaron a surgir los Estados el concepto de soberanía era dogmático, respondía a la supervivencia de los Estados y se trataba de una necesidad política. El concepto clásico de soberanía evoluciona con el avance científico y tecnológico y con la interrelación entre los Estados.

12 Cfr. LÓPEZ VICENTE, Ana Laura, El espacio aéreo y su regulación internacional, http://bit.ly/2a0AvZ0, 04/05/2019, 07:57.

13 Cfr. El espacio aéreo. Competencias estatales y régimen jurídico de la navegación aérea internacional, http:// bit.ly/2a0ATqb, 04/05/2019, 10:23.

14 Cfr. ERDOZAIN, Martin Luis, El Espacio Aéreo y su Regulación Internacional, http://bit.ly/1iMbQuM, 04/05/2019, 17:39.

15 Término jurídico latino (imperǔum) que designaba en la Antigua Roma el poder de mando y castigo, de índole militar, del gobernante sobre los ciudadanos convocados a la guerra y el dominio sobre los territorios conquistados.

16 El dominio o dominium es la posibilidad amparada del derecho de identificar una cosa corporal para obtenerla y tenerla y de ejercer actos sobre ella que impliquen el máximo aprovechamiento de sus ventajas o utilidades. 
Existen restricciones a su ejercicio originadas en el derecho natural y en los compromisos adquiridos con la comunidad mundial. Se amplía su extensión espacial, surge el concepto de cooperación internacional, y varía su contenido jurídico. ${ }^{17}$ Ya en el inicio de la navegación aérea, surgieron posiciones extremas: la de la libertad absoluta del espacio aéreo para que la actividad aeronáutica pudiera desarrollarse sin trabas frente a la doctrina de la soberanía absoluta del espacio aéreo basada en razones políticas, militares, económicas y de seguridad.

La teoría de la soberanía del aire se funda en argumentos relacionados con las necesidades de asegurar la defensa nacional a través del criterio de la soberanía estatal. ${ }^{18}$ Esta posición fue adoptada por normas internas e internacionales y se apoya en el hecho de que la aviación volvía vulnerable las fronteras. Se ha entendido que la primera afirmación de soberanía estatal sobre el espacio aéreo, apareció en Suiza, Estado neutral en la Primera Guerra Mundial, ${ }^{19}$ que, en 1914, como reacción a los vuelos británicos y franceses, declaró que sus derechos de soberanía sobre el espacio aéreo eran ilimitados.

Con el desplazamiento de las aeronaves y su evolución tecnológica, han aparecido nuevas nociones de frontera-superficie y de frontera-volumen. ${ }^{20} \mathrm{El}$ reconocimiento de la plena libertad del espacio aéreo produce la opción por el concepto de frontera-superficie, mientras que el reconocimiento de la soberanía sobre el espacio aéreo agrega al territorio un volumen, posición ésta que es actualmente seguida por los convenios internacionales en la materia y en las legislaciones internas. ${ }^{21}$

El derecho de soberanía ejercido por el Estado sobre el espacio aéreo ubicado encima de su territorio implica la manifestación de diferentes facultades, entre ellas: reglamentar, ordenar la circulación aérea, fijar zonas prohibidas o restringidas, ejercer funciones de policía en el espacio aéreo, entre otras.

El espacio aéreo tiene connotaciones en la esfera del Derecho Privado. ${ }^{22}$ En consecuencia, la proyección de la soberanía sobre el espacio aéreo debe conciliarse con el alcance de los derechos de los propietarios superficiarios respecto del espacio aéreo situado por encima de sus fundos. ${ }^{23}$ Con el surgimiento de la aviación, se agudizaron los conflictos de intereses, y tanto la doctrina, como la legislación y la jurisprudencia fueron buscando amalgamar los derechos de los propietarios

\footnotetext{
17 Cfr. Derecho Espacial, http://bit.ly/2arWB93, 06/05/2019, 07:56.

18 ERDOZAIN, Martin Luis, El Espacio Aéreo y su Regulación Internacional, http://bit.ly/1iMbQuM, op. cit.

$19 C f r$. VELÁZQUEZ ELIZARRARÁS, Juan Carlos, El derecho del espacio ultraterrestre en tiempos decisivos: ¿estatalidad, monopolización o universalidad?, Biblioteca jurídica Virtual del Instituto de Investigaciones Jurídicas de la UNAM, http://bit.ly/29W1mcT, 06/05/2019, 11:18.

20 Cfr. FOLCHI, Mario O., Tratado de Derecho Aeronáutico y Política de la Aeronáutica Civil, http://bit. ly/2aatXcR, 06/05/2019, 16:42.

21 La Constitución y los Tratados Internacionales. Un acercamiento a la interpretación judicial de la jerarquía de las normas y la aplicación de los tratados en la legislación nacional, http://bit.ly/2acqVCE, 06/05/2019, 23:14.

22 Cfr. REGALES CRISTÓBAL, Esteban, Tesis Doctoral, La infraestructura y el Espacio Aéreo como elemento del mercado de transporte, Universidad Autónoma de Barcelona, España, 2015, pp. 122-130.

23 Cfr. Criterios y texto de orientación sobre la reglamentación económica del transporte aéreo internacional, Organización de Aviación Civil Internacional, http://bit.ly/2a25Ye4, 07/05/2019, 08:39.
} 
con la necesidad de utilizar el espacio aéreo como elemento fundamental para la aviación sin que se pusieran trabas.

El hecho de que la propiedad superficiaria se extendiera desproporcionadamente en sentido vertical, con la máxima de que aquél a quien pertenece el suelo, tiene derecho a ejercer sus derechos hasta el cielo, podría llegar a constituir un grave problema para el transporte aéreo. ${ }^{24}$ Por supuesto que con respecto a esta determinación vertical de los derechos de los superficiarios las opiniones fueron antagónicas, extremas y algunas intermedias.

\section{EL CONVENIO DE CHICAGO DE 1944}

Con los avances tecnológicos y el incremento de la actividad aérea, la comunidad internacional se vio en la necesidad de establecer un régimen jurídico para el espacio aéreo, datando sus primeros intentos de principios del siglo pasado. La Segunda Guerra Mundial tuvo una gran influencia en los progresos técnicos del avión y fue necesario resolver problemas técnicos y políticos así como conflictos económicos y jurídicos que se planteaban con el sobrevuelo en tiempos de paz sobre diferentes fronteras y asuntos vinculados a los derechos comerciales.

Es decir, ¿qué acuerdos podrían celebrar los Estados para que los servicios aéreos de uno de ellos pudiesen ingresar en el espacio aéreo de otro? El 7 de diciembre de 1944, se firma en Chicago el mencionado Convenio sobre Aviación Civil Internacional ${ }^{25}$ principal instrumento regulador de la aviación civil, que retoma, adaptando a la época histórica, ideas plasmadas en las Convenciones de París de 1919, de Madrid de 1926 y de La Habana de 1928.

El Convenio se fijó como objetivos: promover el desarrollo ordenado de la aviación civil de postguerra y facilitar el transporte aéreo. Fue fruto de una transacción entre los Estados que predicaban una libertad absoluta en el espacio aéreo, tal el caso de Estados Unidos cuya aeronáutica se había visto fortalecida y que bregaba por un acuerdo multilateral de cielos abiertos (open skies), y otros Estados que reivindicaban soberanía sobre sus espacios aéreos y restricciones a la actividad aérea.

Dicho Convenio consta de 96 Artículos en los que se establecen los privilegios y obligaciones de todos los Estados contratantes, la adopción de normas y métodos recomendados internacionales para regular la navegación aérea, así como recomendaciones a los Estados contratantes para que establezcan servicios e instalaciones de navegación aérea, proponiendo la facilitación del transporte aéreo mediante la reducción de trámites aduaneros y de inmigración.

Como ya se mencionó, el Convenio reconoce en su Artículo $1^{\circ}$ el principio de soberanía plena y exclusiva de todo Estado en el espacio aéreo sobre su territorio, considerando como territorio en su Artículo $2^{\circ}$ las áreas terrestres y las aguas territoriales adyacentes a ellas que se encuentren bajo la soberanía, dominio, protección o mandato de dicho Estado.

24 Cfr. El dominio espacial del Derecho internacional público, http://bit.ly/1MlYelV, 07/05/2019, 12:48.
25 Convenio sobre Aviación Civil Internacional (convenio de Chicago), http://bit.ly/2a5tKqa, op. cit. 
Habida cuenta de que la aviación puede ser utilizada con fines ilícitos, en la década de los 80 se promovió la adopción del Artículo 3 bis que dio lugar a un Protocolo relativo a una Enmienda al Convenio de Chicago. Este artículo se refiere a la violación del espacio aéreo, sin definirla. Esta se produce cuando una aeronave vuela sin someterse a las instrucciones que el Estado detentador de su soberanía ha establecido legítimamente. En su inciso b), dicho Artículo establece entre otras consideraciones que:

Los Estados contratantes reconocen que todo Estado tiene derecho, en el ejercicio de su soberanía, a exigir el aterrizaje en un aeropuerto designado de una aeronave civil que sobrevuele su territorio sin estar facultada para ello, o si tiene motivos razonables para llegar a la conclusión de que se utiliza para propósitos incompatibles con los fines del presente Convenio...."

En efecto; hay varios hechos ilícitos que se producen utilizando aeronaves que sobrevuelan el territorio del Estado, tales el contrabando, el espionaje, el transporte ilegal de personas y sustancias psicotrópicas y estupefacientes entre otros. Uno de los temas más discutidos durante la Conferencia de resultas de la cual se aprobó el citado instrumento, fue el de la concesión recíproca de derechos comerciales en la aviación civil internacional.

A ese respecto, el Artículo $5^{\circ}$ reconoce el derecho de los servicios aéreos internacionales no regulares a penetrar en el territorio de los demás Estados contratantes o sobrevolarlo sin escalas, y a hacer escalas en él con fines no comerciales, sin necesidad de obtener permiso previo, reconociéndoles además la tercera y cuarta libertad del aire, aunque con determinadas reservas. Entre ellas, la posibilidad del Estado sobrevolado de exigir el aterrizaje por razones de seguridad, o que los vuelos se cumplan por determinadas rutas prescriptas.

Por su parte, el Artículo $6^{\circ}$ de dicho Convenio, establece que no podrá explotarse ningún servicio aéreo internacional regular sobre el territorio de un Estado contratante o hacia el interior de éste, sin su consentimiento previo. ${ }^{26}$ Como las discrepancias respecto del otorgamiento sobre derechos aerocomerciales no pudieron resolverse en el propio Convenio de Chicago, se dictaron dos acuerdos suplementarios del mismo: el Acuerdo relativo al tránsito de los servicios aéreos internacionales y el Acuerdo sobre transporte aéreo internacional. En la actualidad, el primero de los Acuerdos nombrados cuenta con 122 ratificaciones y el segundo con 11 .

En el Acuerdo relativo al tránsito, se expresa el concepto de primera libertad del aire, conocida como derecho de sobrevuelo y el de segunda libertad del aire o derecho de escala técnica que se ejerce para reabastecerse de combustible, efectuar un aterrizaje de emergencia o realizar reparaciones esenciales imprevistas.

Por su parte, el Acuerdo sobre transporte aéreo internacional o acuerdo de las cinco libertades hacen referencia a los derechos de tráfico tomados en el

$\overline{26}$ Cfr. Convenio relativo al tránsito de los servicios aéreos internacionales, http://bit.ly/2ammnvq, 07/05/2019, 22:43. 
sentido de derechos de acceso a los mercados, consagra las cinco libertades del aire, agregando a las dos mencionadas del Acuerdo de tránsito, las llamadas tercera, cuarta y quinta libertad. De la combinación de diferentes supuestos, han surgido otras libertades, que se han denominado sexta, séptima (cabotaje), ${ }^{27}$ octava libertad o cabotaje consecutivo, novena libertad o cabotaje autónomo. ${ }^{28} \mathrm{~A}$ consecuencia de la poca aceptación internacional del Acuerdo de Transporte Aéreo, los Estados regularon sus respectivos derechos de explotación por la vía de Convenios bilaterales.

En los últimos tiempos es frecuente volver a utilizar la expresión cielos abiertos en tanto concepto aeropolítico, retomando la posición que ya fuera sustentada por los Estados Unidos en las reuniones previas a la firma del Convenio de Chicago. ${ }^{29}$ Las discusiones acerca de las ventajas y desventajas de la llamada política de cielos abiertos, han sido numerosas. Los Convenios de cielos abiertos posibilitan la explotación de los servicios aéreos libremente por parte de las líneas aéreas designadas por los Estados signatarios, en ambas direcciones entre los respectivos territorios y sin restricciones respecto del tipo de aeronaves, capacidad ofrecida, frecuencias y tarifas.

Existen en la actualidad Acuerdos multilaterales que adoptan la política de cielos abiertos, tal el caso del Convenio multilateral en el marco del Foro de Cooperación Económica Asia-Pacífico (APEC) suscrito entre Estados Unidos, Brunei, Chile, Nueva Zelanda y Singapur. ${ }^{30}$ La integración regional ha dado lugar a una nueva normativa aeronáutica, la Comunitaria de la Unión Europea y los Acuerdos regionales en América. Se está produciendo un cambio en la negociación sobre los Acuerdos relacionados con la explotación de los servicios aéreos, ya que se va hacia una negociación en bloque de los derechos aerocomerciales por parte de las distintas regiones, y el bilateralismo ya no será entre Estados sino entre bloques regionales.

Con el fin de desarrollar los principios y técnicas de la navegación aérea internacional, fomentar la organización y el desenvolvimiento del transporte aéreo internacional, promoviendo en general, el desarrollo de la aeronáutica civil internacional en todos sus aspectos, el Artículo 43 del Convenio de Chicago crea un organismo denominado Organización de Aviación Civil Internacional (OACI). ${ }^{31}$

Dicha organización es una agencia especializada de las Naciones Unidas, integrada por 188 Estados, cuya sede se encuentra en la ciudad de Montreal, Canadá. Está compuesta por una asamblea, un consejo y otros órganos que se han estimado necesarios. Su principal objetivo es lograr el desarrollo seguro y ordenado de la aviación civil internacional en todo el mundo. Existe un compromiso

\footnotetext{
27 Transporte público aeronáutico entre puntos de un mismo país.

28 Convenios bilaterales en materia de transporte aéreo: las libertades del aire, http://bit.ly/2adljLe, 08/05/2019, 10:18.

29 Cfr. Dr. FOLCHI, Mario O., La Convención de Chicago de 1944 y la política de cielos abiertos, http://bit. ly/2a5Ov54, 08/05/2019, 16:18.

30 Cfr. FREYRE LAYZEQUILLA, Augusto, Convenios aéreos y el contexto normativo internacional, http:// bit.ly/2acoCSA, 08/05/2019, 22:29.

31 Cfr. Organización de Aviación Civil Internacional, http://bit.ly/1CdTlle, 09/05/2016, 08:17.
} 
de los Estados miembros del Convenio de Chicago, establecido en el Artículo 37 en cuanto se comprometen a colaborar, a fin de lograr el más alto grado de uniformidad posible en las reglamentaciones, normas, procedimientos y organizaciones relativos a las aeronaves, personal, aerovías y servicios auxiliares, en todas las cuestiones en que tal uniformidad facilite y mejore la navegación aérea.

A tales efectos la OACI ha adoptado y enmendado en su caso, las llamadas Normas y Métodos recomendados internacionales, conocidos como anexos al Convenio sobre Aviación Civil Internacional, los cuales tratan de temas diversos, tales como: servicios de tránsito aéreo, operación de aeronaves, facilitación, búsqueda y salvamento, aeronavegabilidad, transporte sin riesgos de mercaderías peligrosas por vía aérea, entre estos anexos tienen un mecanismo sui generis para su entrada en vigencia internacional, el que se encuentra especificado en el convenio. ${ }^{32}$ Por otra parte, a efectos de cumplir con el objetivo de la uniformidad entre las legislaciones de los Estados, nuestro país ha incorporado los anexos al orden interno.

Con relación a los tratados multilaterales distinguimos los principales:

Tratado multilateral general.

El Convenio de Chicago de 7 de diciembre de 1944 que contiene dos grandes categorías de disposiciones: las reglas que dieron lugar a la OACI y las disposiciones dirigidas a reglamentar la navegación y ciertos aspectos del transporte aéreo.

Convenios multilaterales sobre determinadas materias.

- Convenio de Varsovia de 12 de octubre de 1929 para la unificación de determinadas reglas relativas al transporte aéreo internacional, modificado por los protocolos firmados en La Haya el 28 de septiembre de 1955 y en la ciudad de Guatemala el 8 de marzo 1971.

- Convenio de Roma sobre embargo preventivo de aeronaves de 29 de mayo de 1933.

- Convenio de Bruselas de 1938 sobre Asistencia y Salvamento (no tuvo vigencia).

- Convenio de Ginebra de 19 de junio de 1948 sobre reconocimiento internacional de derechos sobre aeronaves.

- Convenio de Roma de 7 de octubre de 1952 relativo a la unificación de ciertas reglas referentes a los daños causados por la aeronave a terceros en superficie.

- Convenio de Guadalajara de 18 de septiembre de 1961, para la unificación de ciertas reglas relativas al transporte aéreo internacional realizado por quien no sea el transportista contractual.

- Convenio de Tokio de 14 de septiembre de 1963 sobre infracciones y ciertos actos sometidos a bordo de aeronaves.

- Convenio de París de 10 de julio de 1967 sobre procedimiento aplicable para el establecimiento de las tarifas de los servicios aéreos regulares.

$\overline{32}$ Cfr. Convenio sobre Aviación Civil Internacional, Anexos 1 a 18, http://bit.ly/2aeNc5s, 09/05/2019, 10:48. 
- Acuerdo de Montreal de 4 de marzo de 1966 relativo a los límites de responsabilidad del convenio de Varsovia y del protocolo de La Haya.

- Convenio de La Haya de 16 de diciembre de 1970 para la represión del apoderamiento ilícito de aeronaves.

- Convenio de Montreal de 23 de septiembre de 1971 para la represión de actos ilícitos contra la seguridad de la Aviación Civil.

Acuerdos regionales.

Por lo que respecta a los Convenios que no tienen un alcance mundial sino que afectan sólo a determinadas regiones, citamos:

- Acuerdo de París de 30 de abril de 1956 sobre derechos comerciales de servicios aéreos no regulares europeos.

- Acuerdo de 13 de diciembre de 1960 por el que determinados países europeos establecieron una organización común encargada de regular la circulación aérea.

- Tratado de Yaundé de 28 de marzo de 1961 para la explotación del transporte aéreo por una sociedad común (Air Afrique) entre determinados países africanos.

\section{INSTITUCIONES RELEVANTES EN LA NORMATIVIDAD AERONÁUTICA INTERNACIONAL}

FAA (Federal Aviation Administration). ${ }^{33}$ La Administración Federal de Aviación (FAA) es la autoridad aeronáutica nacional de los Estados Unidos. Como agencia del Departamento de Transporte de los Estados Unidos, tiene autoridad para regular y supervisar todos los aspectos de la aviación civil estadounidense. Su misión es contar con sistema aeronáutico más seguro y eficiente del mundo.

IATA (Asociación Internacional de Transportes Aéreos). ${ }^{34}$ La IATA busca ayudar a las líneas aéreas simplificando los procesos e incrementando la conveniencia del flujo financiero de sus ingresos mientras reduce costos y aumenta la eficiencia. Este instrumento para la cooperación entre aerolíneas, promueve la seguridad, fiabilidad, confianza y negocios en el transporte aéreo en beneficio de sus accionistas privados. Puede pertenecer a la IATA cualquier compañía aérea que tenga la posibilidad de operar un servicio aéreo regular internacional por el Gobierno de un Estado que pertenezca a la OACI.

Los objetivos y servicios que ofrece la IATA son:

- Ofrecer beneficios a todas las partes involucradas en el comercio aéreo.

- Para los consumidores: Simplifica los procesos de viaje y transporte, mientras mantiene los costos bajos.

- $\quad$ IATA permite que las aerolíneas operen de manera segura, eficiente y económica, bajo reglas definidas.

\footnotetext{
33 Cfr. FAA, http://www.faa.gov/, 09/05/2019, 15:48.

34 Cfr. Asociación Internacional de Transportes Aéreos, http://bit.ly/QHyc3i, 09/05/2019, 20:12.
} 
- IATA sirve de intermediario entre el pasajero, los agentes de carga y las aerolíneas.

- Para los gobiernos, IATA busca asegurar que ellos puedan estar bien asesorados en las complejidades de la industria de la aviación.

DGAC. (Dirección General de Aeronáutica Civil). ${ }^{35}$ La Dirección General de Aeronáutica Civil tiene como objetivo, asegurar que el transporte aéreo mexicano participe en el proceso de crecimiento sostenido y sustentable, que contribuya al bienestar social, al desarrollo regional y a la generación de empleos, apoyando la confirmación de una sociedad mejor integrada y comunicada. El 16 de octubre del 2019 se promulgo el Decreto de la creación de la Agencia Federal de Aviación Civil, la cual transforma a la cita dirección como un organismo desconcentrado dependiente de la Secretaría de Comunicaciones y Transporte

\section{CONCLUSIÓN}

Ni la Convención de París de 1919 ni la vigente Convención de Chicago de 1944 contienen una definición y delimitación precisas del espacio aéreo. De acuerdo con estas disposiciones, el espacio aéreo medido horizontalmente tiene la misma extensión longitudinal que la suma del terrestre y el marítimo sometido a la soberanía estatal, abarcando, por tanto, la tierra, las aguas interiores y el mar territorial. Pero, si de acuerdo con ello, el límite horizontal del espacio aéreo resulta suficientemente preciso (sobre todo una vez que se ha conseguido fijar, en el marco de la III Conferencia de las Naciones Unidas sobre el Derecho del Mar y en el nuevo Convenio de 1982, una extensión uniforme para el mar territorial de doce millas marinas), no ocurre lo mismo respecto a su límite vertical, es decir, con relación a la delimitación del espacio aéreo con el espacio ultraterrestre.

La indeterminación del límite vertical del espacio aéreo ha suscitado numerosas teorías a nivel doctrinal:

Parte de la doctrina entiende que la fijación de tales límites podría deducirse de la definición del término "aeronave", contenida en los Anexos de la Convención de Chicago, comprendiendo el espacio aéreo hasta donde un aparato de vuelo puede sustentarse en la atmósfera merced a las reacciones del aire.

Otro sector doctrinal ha estimado que los autores de la Convención no tuvieron la intención de establecer una delimitación del espacio aéreo. Además, los criterios técnicos introducidos por dicha Convención en relación con la capacidad aerodinámica de los aparatos de vuelo no fueron criterios estables: la tecnología fue paulatinamente ampliando esa capacidad aerodinámica, de modo que la frontera técnica del espacio aéreo ha ido extendiéndose en proporción al avance operativo de la aeronáutica.

Hoy día subsiste la cuestión de determinar el límite vertical del espacio aéreo o, lo que es lo mismo, la delimitación entre el espacio aéreo y el espacio ultraterrestre, lo que no ha impedido, sin embargo, que ambos espacios hayan

$\overline{35}$ Cfr. Dirección General de Aeronáutica Civil, http://bit.ly/1nikpOe, 09/05/2019, 22:47. 
sido perfectamente definidos en cuanto a su régimen jurídico: el espacio aéreo sujeto a soberanía estatal plena y exclusiva, y el espacio exterior o ultraterrestre dominado por el principio de no apropiación nacional.

\section{BIBLIOGRAFÍA}

ÁLVAREZ, José Francisco, Capacidades, libertades y desarrollo: Teorías Políticas Contemporáneas, Editorial Tirant Lo Blanch, Valencia, España, 2001.

Comisión sobre la Utilización del Espacio Ultraterrestre con Fines Pacíficos, http:// bit.ly/2am29SM, 03/05/2019, 19:08.

Constitución Política de los Estados Unidos Mexicanos, Última reforma publicada DOF, 26 de febrero del 2013, http://bit.ly/1KpWTdj, 11/04/2015

Convenio sobre Aviación Civil Internacional (convenio de Chicago), http://bit. ly/2a5tKqa, 03/05/2016, 11:43.

Convenio sobre Aviación Civil Internacional (convenio de Chicago), http://bit. ly/2a5tKqa, op. cit.

Convenio relativo al tránsito de los servicios aéreos internacionales, http://bit. ly/2ammnvq, 07/05/2019, 22:43.

Convenios bilaterales en materia de transporte aéreo: las libertades del aire, http:// bit.ly/2adljLe, 08/05/2019, 10:18.

Convenio sobre Aviación Civil Internacional, Anexos 1 a 18, http://bit.ly/2aeNc5s, 09/05/2019, 10:48.

COPUOS, http://www.unoosa.org/pdf/transcripts/copuos/COPUOS, 03/05/2019, $11: 30$.

Criterios y texto de orientación sobre la reglamentación económica del transporte aéreo internacional, Organización de Aviación Civil Internacional, http:// bit.ly/2a25Ye4, 07/05/2019, 08:39.

DABIN, Jean, Doctrina General del Estado, Instituto de Investigaciones Jurídicas, UNAM, primera edición, México, 2003.

Derecho Espacial, http://bit.ly/2arWB93, 06/05/2019, 07:56.

El Espacio Aéreo y su Régimen Jurídico, http://bit.ly/2aabey5, 03/05/2016, 08:27.

El Espacio Aéreo. Competencias estatales y régimen jurídico de la navegación aérea internacional, http://bit.ly/2a0ATqb, 04/05/2019, 10:23.

El dominio espacial del Derecho internacional público, http://bit.ly/1MlYelV, 07/05/2019, 12:48.

ERDOZAIN, Martin Luis, El Espacio Aéreo y su Regulación Internacional, http:// bit.ly/1 iMbQuM, 04/05/2019, 17:39.

ESPARZA, Rafael R., La Aviación. Secretaría de Comunicaciones y Transportes, México, 1987.

FAA, http://www.faa.gov/, 09/05/2019, 15:48.

FOLCHI, Mario O., Tratado de Derecho Aeronáutico y Política de la Aeronáutica Civil, http://bit.ly/2aatXcR, 06/05/2019, 16:42.

FOLCHI, Mario O., La Convención de Chicago de 1944 y la política de cielos abiertos, http://bit.ly/2a5Ov54, 08/05/2019, 16:18. 
FREYRE LAYZEQUILLA, Augusto, Convenios aéreos y el contexto normativo internacional, http://bit.ly/2acoCSA, 08/05/2019, 22:29.

La administración pública en los estados y reflexiones sobre el federalismo. Convergencia: Revista de Ciencias Sociales, 2009.

La Constitución y los Tratados Internacionales. Un acercamiento a la interpretación judicial de la jerarquía de las normas y la aplicación de los tratados en la legislación nacional, http://bit.ly/2acqVCE, 06/05/2019, 23:14.

LINARES, Antonio, Régimen Jurídico del Espacio Aéreo, http://bit.ly/2a9hcw9, 03/05/2016, 22:49.

LÓPEZ VICENTE, Ana Laura, El espacio aéreo y su regulación internacional, http://bit.ly/2a0AvZ0, 04/05/2019, 07:57.

OLIVAR ANGERAMI, Ignacio Augusto, Espacio Ultraterrestre y sus actores, una relación dialéctica, Tesis de Grado correspondiente a la Licenciatura en Relaciones Internacionales, Facultad de Ciencias Humanas, Universidad Nacional del Centro de la Provincia de Buenos Aires, 2012.

Organización de Aviación Civil Internacional, http://bit.ly/1CdTlle, 09/05/2016, 08:17.

ORTIZ AHLF, Loretta, Derecho Internacional Público, University Press Oxford, tercera edición, México, 2004, pp. 105-108.

REGALES CRISTÓBAL, Esteban, Tesis Doctoral, La infraestructura y el Espacio Aéreo como elemento del mercado de transporte, Universidad Autónoma de Barcelona, España, 2015, pp. 122-130.

Tratados y principios de las Naciones Unidas sobre el Espacio Ultraterrestre, ONU, http://bit.ly/2a0yY1D, 03/05/2019, 13:39.

VELÁZQUEZ ELIZARRARÁS, Juan Carlos, El derecho del espacio ultraterrestre en tiempos decisivos: ¿estatalidad, monopolización o universalidad?, Biblioteca jurídica Virtual del Instituto de Investigaciones Jurídicas de la UNAM, http://bit.ly/29W1mcT, 06/05/2019, 11:18. 